\section{Paediatric crossword puzzle 35}

Manouri P Senanayake ${ }^{1,2}$, A S Athapathu ${ }^{2}$

Sri Lanka Journal of Child Health, 2017; 46(2): 195

DOI: http://dx.doi.org/10.4038/sljch.v46i2.8284

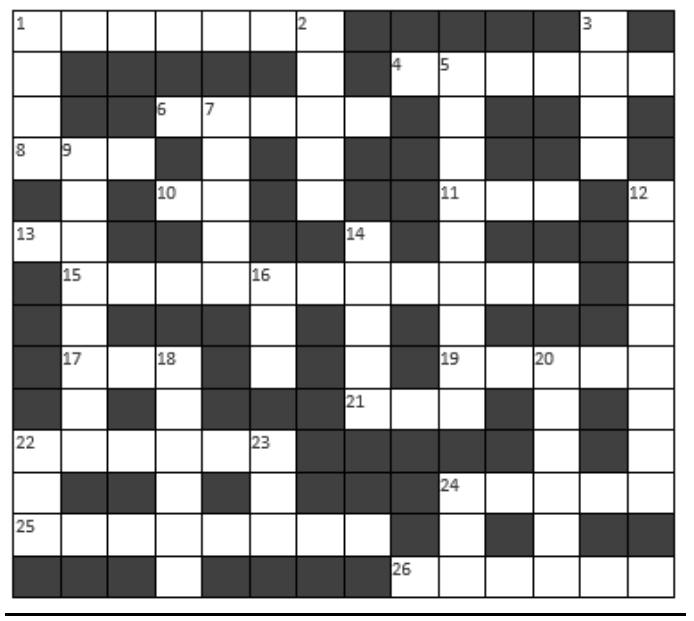

\section{ACROSS}

1. Physical sign suggesting repeated anal penetration

4. At risk of Vitamin $B_{12}$ deficiency

6. Helped by melatonin

8. Heart condition causing appearance of cyanosis after birth (abbrv)

10. Household equipment which reduces reading skills and increases risk of ischaemic heart disease

11. Hormonal investigation in hypercholesterolemia

13. Foetus affected by complete androgen insensitivity syndrome

15. Surgical procedure that helps with self catheterization

17. Abnormal downward pull of the trachea

19. Distortion in size is a feature in In Wonderland syndrome

21. _ _ _ _ denafil reduces pulmonary hypertension

22. Very rare tumour in the heart

24. Benign skin lesions on new-born's face

25. Anatomical structure used in the Mitrafanoff procedure

26. Affected organ of prognostic value in Henoch Schonlein Purpura

\section{DOWN}

1. Caused by papilloma virus

2. Ethambutol causes inability to see ............

3. Antibacterial always prescribed in combination

5. Abuse caused by domestic violence

7. Organ most commonly associated with internal haemangioma

9. Measured in sleep studies

12. Source of Vitamin $B_{12}$

14. Interventions used to correct talipes

16. Stimming is seen in (abbrv)

18. Personal protective equipment that should be the last to be discarded

20. Prebiotic properties found in onion, garlic and leeks are contained in

22. Left to right shunting of blood causes increased diameter on imaging (abbrv)

23. Former term for attention deficit hyperactivity disorder (abbrv)

24. Imaging modality for diagnosing soft tissue lesions (abbrv)

${ }^{1}$ Department of Paediatrics, Faculty of Medicine, Colombo, ${ }^{2}$ Lady Ridgeway Hospital for Children, Colombo 